\title{
Parametric Analysis of Forces and Stresses in Superconducting Quadrupole Sector Windings
}

\author{
P. Fessia, F. Regis, and E. Todesco
}

\begin{abstract}
This paper presents a review of the existing analytical approximations of the magnetic field of a sector winding quadrupole, evaluating to which extent these formulae are usable as a first estimation of the resultants of the electromagnetic forces and related mechanical stresses produced. The distribution of the forces has been investigated to better understand the stress provided to the structure containing the windings and the compression effect induced on the coil mid plane. Therefore, the study has been carried out setting the magnet current at its critical value, analyzing forces and stresses as functions of the geometrical layout and of the magnetic gradient as well. In the last part the effect of an iron yoke on the magnetic field and forces is presented.
\end{abstract}

Index Terms-Current density, stress, superconducting magnets.

\section{INTRODUCTION}

I $\mathrm{N}$ superconducting quadrupoles, e. $\mathrm{m}$. forces and associated stresses of the order of $100 \mathrm{MPa}$ are generated by the interaction of the cable current with the magnetic field. Two main issues have to be analysed: first, a mechanical structure must be envisaged to contain these forces and limit the cable movements during the magnet ramp. Secondly, the stresses inside the coil must not exceed the limits beyond which insulation can start creeping and the superconductor properties are degraded. This second aspect is critical for the $\mathrm{Nb}_{3} \mathrm{Sn}$, which according to some cable measurements cannot tolerate compressive stresses larger than $\sim 150 \mathrm{MPa}[1]$.

In this paper we aim at analyzing how forces and stresses depend on the quadrupole aperture, on the width of the coil, and on the superconducting material. We used a simplified coil layout made up of a sector of inner radius $r_{\mathrm{i}}$, radial width $w$ and an angular extension $\alpha_{0}=30^{\circ}$ (thus canceling the first allowed field harmonic), fed by a uniform current density $j$ (see Fig. 1). This simple geometry has the advantage of being closer to a real coil rather than the classical $\cos 2 \theta$, whilst still allowing an analytical approach.

In [2], it has been shown that a similar lay-out well represents, from e. m. point of view, several quadrupoles based on the shell geometry that have been built in the last 30 years.

The main steps carried out in this analysis have been:

Manuscript received August 28, 2006. This work was supported by the European Community-Research Infrastructure Activity under the FP6 "Structuring the European Research Area" programme (CARE, contract number RII3-CT2003-506395). The work of F. Regis was supported by ASP (Associazione per lo Sviluppo Tecnologico e Scientifico del Piemonte) and Compagnia di San Paolo, Turin, Italy.

The authors are with the Accelerator Technology Department, CERN, Geneva 23, 1211 CH Switzerland (e-mail: paolo.fessia@cern.ch).

Digital Object Identifier 10.1109/TASC.2007.898523

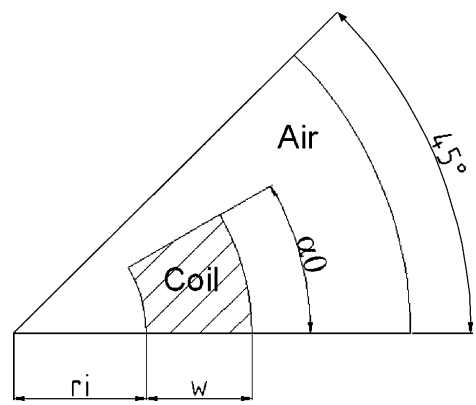

Fig. 1. Quadrupole sector coil layout.

- Estimate analytically using the formalism developed in [3]-[5] the electromagnetic forces and the induced stresses in the sector coil as a function of $w$ and $r_{i}$ for a given $j$. A similar approach has been developed for the dipoles using a $\cos \theta$ model in [6], [7]. In particular, we will focus on the definition of the position and of the value of the maximum compressive stress on the coil mid plane.

- Substitute $j$ with the critical current density relative to that coil lay-out and to that superconductor (either $\mathrm{Nb}-\mathrm{Ti}$ or $\mathrm{Nb}_{3} \mathrm{Sn}$ ), and evaluate the peak stress as a function of the obtained gradient.

- Further develop the formulae for the critical current density in presence of an iron yoke, revising the peak stress behavior in this new condition.

The study is based on an analytical approximation cross-checked with a two-dimensional finite element model coded in ANSYS of one quadrupole octant (see Fig. 1). The magnetic model of the winding built in ANSYS is surrounded by air.

\section{ForCES AND STRESSES FOR A UNIFORM CURRENT DENSITY}

\section{A. Lorentz Forces}

As first step, the profiles of the magnetic field in the aperture, in the coil, and outside the coil have been computed using an analytical approach [3]-[5] and the numerical model. The results show that

- There is a good agreement between the numerical and the analytical estimate of the magnetic field both in the aperture and outside the coil.

- The agreement is worse inside the coil, the error being dependent on the angular dimension of the sector coil and increasing as $\alpha_{0}$ approaches $45^{\circ}$.

Despite of the aforementioned discrepancy, the analysis of the magnetic energy $U_{m}$ reveals that the analytical approximation 


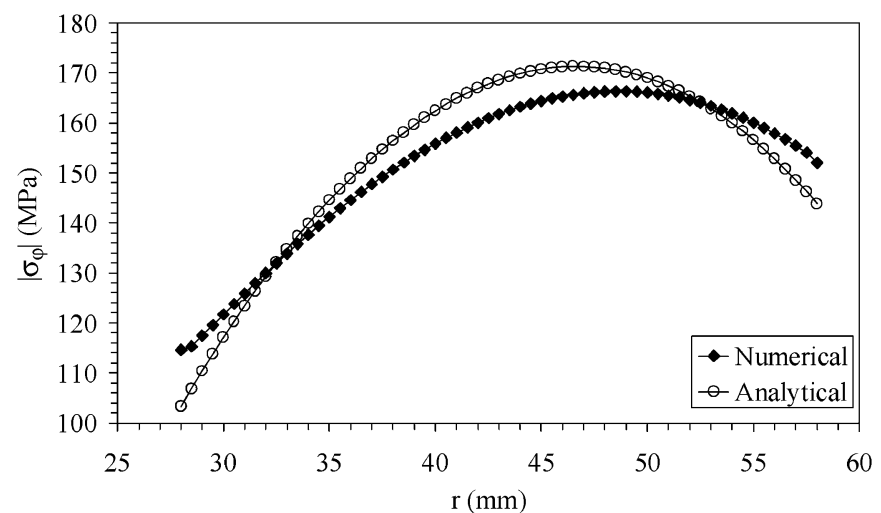

Fig. 2. Azimuthal stress distribution (compression) on coil mid plane $\sigma_{\varphi}(r)$ for a coil with inner radius $r_{i}$ of $28 \mathrm{~mm}$ and a width $w$ of $30 \mathrm{~mm}$, for a current density $j=1000 \mathrm{~A} / \mathrm{mm}^{2}$.

agrees with the numerical one within $4 \%$. A similar result is found for the forces integrated over the coil.

One can conclude that notwithstanding the discrepancy between numerical and analytical results relative to the field distribution inside the coil, the analytical approach provides a very good estimation of the coil magnetic energy and of the coil forces. However, the analytical formulation cannot be used to determine the coil peak field (and therefore the critical current) since it does not provide adequate estimation of the magnetic field inside the coil. This estimate will be carried out using a numerical method as outlined in [2].

\section{B. Stresses on Coil Mid Plane and Collar Contact Edge}

The equations for the azimuthal compressive stress on the coil mid plane $\sigma_{\varphi}(r)$ and of the radial compressive stress on the external profile of the coil $\sigma_{r}(\varphi)$ can be evaluated through balancing the forces acting on an infinitesimally small coil element not considering the shear effect. Integrating the differential equations given by the force balance, we can get:

$$
\begin{aligned}
\sigma_{\varphi}(r)= & -\frac{j^{2} \mu_{0}}{r^{2}}\left[r^{4}-r_{i}^{4}+4 r^{4} \ln \left(\frac{r_{i}+w}{r}\right)\right] \\
& \times \frac{\cos \alpha_{0} \sin ^{3} \alpha_{0}}{\pi} \\
\sigma_{r}(\varphi)= & -\frac{j^{2} \mu_{0}}{\left(r_{i}+w\right)^{2}} f\left(r_{i}^{4}, w^{4}, \alpha_{0}, \varphi\right) \frac{\sin 2 \alpha_{0}}{36 \pi}
\end{aligned}
$$

where $f$ is a function involving geometrical parameters. In these equations, stresses are in $\mathrm{Pa}$, lengths in $\mathrm{m}$, current density in $\mathrm{A} / \mathrm{m}^{2}$. In this analytical approximation higher order terms in $r$, $w$, and shear stresses are neglected. In Fig. $2 \sigma_{\varphi}(r)$ as provided by (1) is compared with the numerical results of a model with an isotropic material, showing a very good agreement, with a maximum error of 5\%, for an inner coil of $28 \mathrm{~mm}$ radius and $30 \mathrm{~mm}$ width. A similar agreement is found also for the radial stress.

\section{Dependence on Material Properties}

The previous analysis shows that the analytical approach is a good approximation for the stresses distribution in an isotropic material. In order to verify the applicability in case of real superconducting windings, where some anisotropy is present due

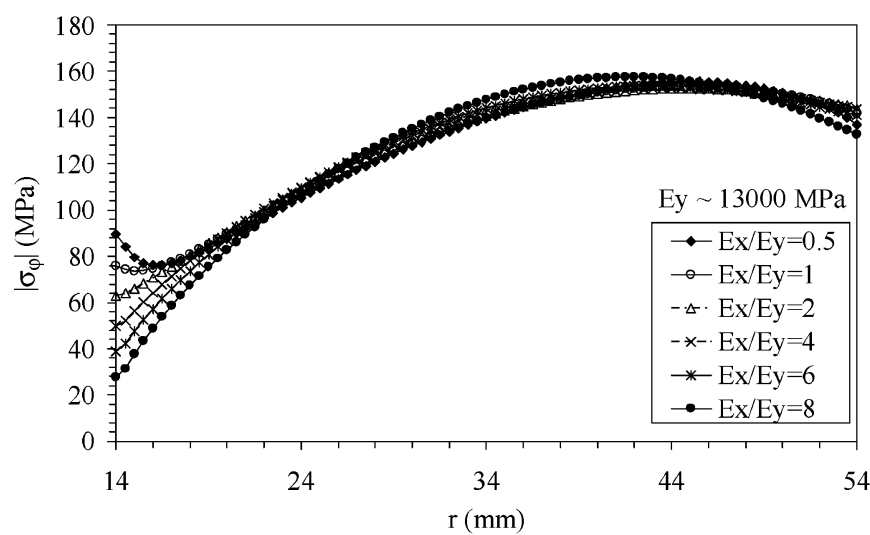

Fig. 3. Dependence of the azimuthal stress distribution on coil mid plane $\sigma_{\varphi}(r)$ on the material anisotropy for a case with inner radius $r_{i}=14 \mathrm{~mm}$, coil width $w=40 \mathrm{~mm}$, and current density $j=1000 \mathrm{~A} / \mathrm{mm}^{2}$.

to the structure of the cable and insulation, simulations have been carried out setting an azimuthal Young's modulus $E$ of about $13 \mathrm{GPa}$ and increasing the modulus in the radial direction. Notwithstanding a relevant difference in the inner radius values (see Fig. 3), only minor discrepancies are found for the maximum stress on the mid plane, that is the quantity of interest. A weak dependence of the position of the maximum stress on the material anisotropy is also observed. We conclude that the analytical approximation is also suitable for anisotropic materials as measured for the superconducting coils.

\section{LORENTZ FORCES AND STRESSES AT CRITICAL CURRENT DENSITY}

In the previous equations, stresses are proportional to the square of the current density $j$. Indeed, in a superconducting quadrupole $j$ is limited by the critical current density $j_{c}$, i.e. the current corresponding to a peak field on the critical surface. In this section we will compute stresses when the current density is set at its critical value. In order to estimate the critical current, following the approach of [2] we define the lay-out parameters

$$
\begin{aligned}
G & =j \gamma\left(r_{i}, w\right) \\
B_{p} & =j \beta\left(r_{i}, w\right)
\end{aligned}
$$

For a sector coil of $\alpha_{0}=30^{\circ}$ one has

$$
\gamma=\gamma_{0} \ln \left(1+\frac{w}{r_{i}}\right)
$$

and $\beta$ is well fit by an empirical expression

$$
\beta=r_{i} \gamma_{0} \ln \left(1+\frac{w}{r_{i}}\right)\left(a_{-1} \frac{r_{i}}{w}+1+a_{1} \frac{w}{r_{i}}\right)
$$

where $a_{-1}, a_{1}$ and $\gamma_{0}$ are constants related to the $30^{\circ}$ lay-out. The $\mathrm{Nb}$-Ti critical surface is fitted by a linear relation

$$
j_{c}=c \kappa\left(B_{c 2}^{*}-B\right), \quad B<B_{c 2}^{*}
$$

where the equation is written for the engineering current density $j$, i.e. the current divided by the area of the insulated conductor. 
TABLE I

PARAMETERS OF THE CRITICAL SURFACE FITS FOR Nb-Ti AND $\mathrm{Nb}_{3} \mathrm{Sn}$

\begin{tabular}{lllll}
\hline \hline & \multicolumn{3}{c}{ NB-TI } & \multicolumn{2}{c}{$\mathrm{NB}_{3} \mathrm{SN}$} \\
Temp. $(\mathrm{K})$ & 4.2 & 1.9 & 4.2 & 1.9 \\
$\mathrm{~B}_{\mathrm{c} 2}^{*}(\mathrm{~T})$ & 10 & 13 & 22.3 & 26.3 \\
$\mathrm{c}\left(\mathrm{A} /\left(\mathrm{T} \mathrm{mm}^{2}\right)\right)$ & 600 & 600 & 3400 & 2500 \\
\hline \hline
\end{tabular}

The fitting parameter $c$ is the slope of the critical surface, and $B_{\mathrm{c} 2}^{*}$ is the critical field according to the fit (see Table I), underestimating the actual one by around $10 \%$. The filling ratio $\kappa$ is a dilution factor taking into account the presence of copper, voids and insulation in the coil; $\kappa$ is equal to 1 for a coil made only of superconductor and in the real case is in a range between 0.23 and 0.33 . Introducing the critical surface fit in (3)-(7), we obtain the expression for the critical current of a sector winding of inner radius $r_{\mathrm{i}}$ and width $w$ made of $\mathrm{Nb}$-Ti conductor.

$$
j_{c, N b-T i} \sim \frac{\kappa c B_{c 2}^{*}}{1+\kappa c r_{i}\left(a_{-1} \frac{r_{i}}{w}+1+a_{1} \frac{w}{r_{i}}\right) \gamma_{0} \ln \left(1+\frac{w}{r_{i}}\right)}
$$

and the critical gradient $G_{\mathrm{c}}=j_{\mathrm{c}} \gamma$ can be obtained by multiplying by (5).

For the $\mathrm{Nb}_{3} \mathrm{Sn}$ we use the fit proposed in [2]

$$
j_{s c}=c\left(\frac{B_{c 2}^{*}}{B}-1\right) \quad B<B_{c 2}^{*}
$$

which is accurate within $5 \%$ with respect to the Summer's approximation [8] between 11 and $17 \mathrm{~T}$ at $1.9 \mathrm{~K}$, and has the advantage of allowing an explicit solution for the critical current

$$
j_{c, N b 3 S n}=\frac{\kappa c}{2}\left(\sqrt{\frac{4 B_{c 2}^{*}}{\beta \kappa c}+1}-1\right)
$$

with $\beta$ given by (6), and similarly for the critical gradient. The fitting parameters $c$ and $B_{\mathrm{c} 2}^{*}$ for both materials at 4.2 and $1.9 \mathrm{~K}$ are listed in Table I.

We then evaluate from (1) the position along the mid plane where the compressive stress is the maximum in the coil; we then substitute the critical current expressions (8) or (10). We iterate this computation for different inner radii and different coil widths, evaluating both the maximum stress and the critical gradient obtained by each configuration. Quadrupole apertures between $r_{\mathrm{i}}=20 \mathrm{~mm}$ and $r_{\mathrm{i}}=60 \mathrm{~mm}$ have been analysed; coil widths $w$ have been chosen between $5 \mathrm{~mm}$ and the value $w=2 r_{\mathrm{i}}$ corresponding to a saturation of the critical gradient to its maximum value.

Results are shown in Figs. 4 and 5 for $\mathrm{Nb}-\mathrm{Ti}$ and $\mathrm{Nb}_{3} \mathrm{Sn}$ respectively. In both cases, we also evaluated a few lay-outs through a numerical simulation to verify the agreement with the analytical approach, which is shown to be very good.

The stress increases for larger apertures: in the largest analysed case $r_{\mathrm{i}}=60 \mathrm{~mm}$ the stress is just below $90 \mathrm{MPa}$ for the $\mathrm{Nb}-\mathrm{Ti}$ and about $150 \mathrm{MPa}$ for $\mathrm{Nb}_{3} \mathrm{Sn}$, which is the maximum acceptable stress in magnet design. The dependence on the coil thickness $w$ is more involved: for the $\mathrm{Nb}$ - Ti in large

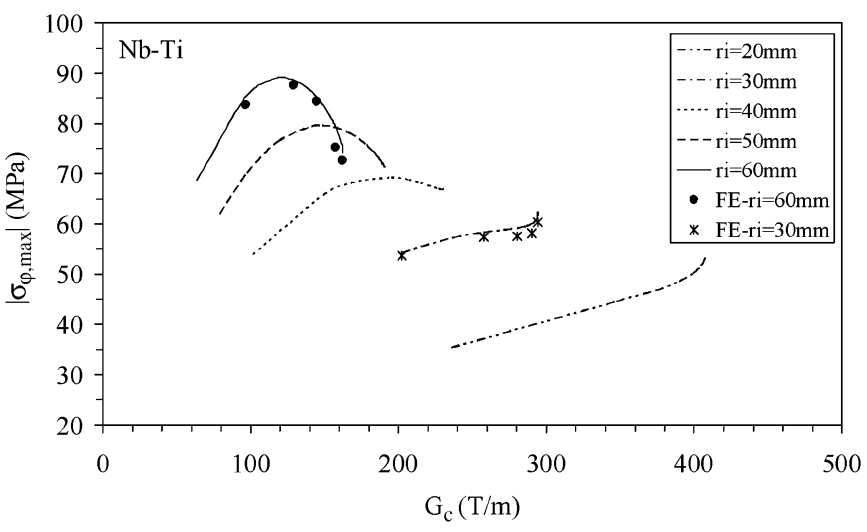

Fig. 4. Maximum azimuthal compressive stress on coil mid plane for different coil layouts considering a Nb-Ti cable (constant aperture radius). $\mathrm{T}=1.9 \mathrm{~K}$.

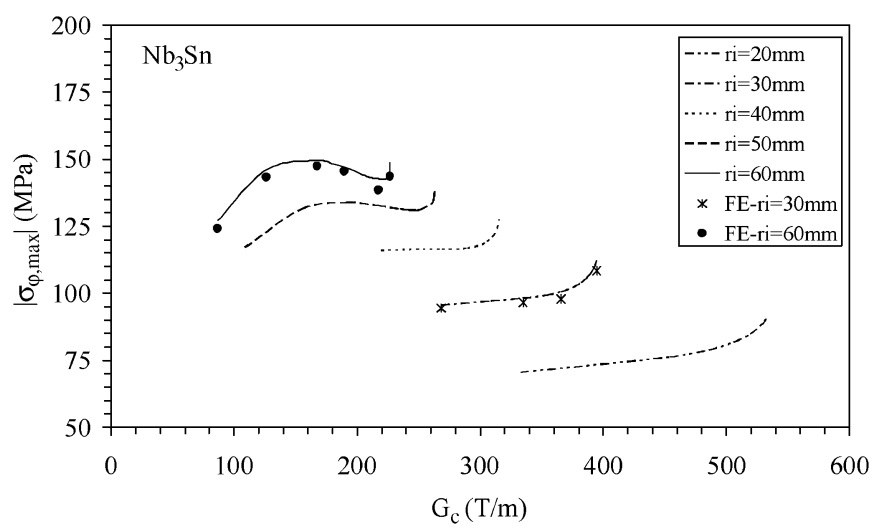

Fig. 5. Maximum azimuthal compressive stress on coil mid plane for different coil layouts considering a $\mathrm{Nb}_{3} \mathrm{Sn}$ cable (constant aperture radius). $\mathrm{T}=4.2 \mathrm{~K}$.

apertures $\left(r_{\mathrm{i}}>30 \mathrm{~mm}\right)$ the peak stress does not increase monotonically with $G_{\mathrm{C}}$ (which is a function of $w$ ): a maximum is found for a given $G_{c}$, after having reached that point the stress decreases as $G_{\mathrm{c}}$ increases (since $w$ increases faster then $G_{\mathrm{c}}$ ). This behavior appears also for the $\mathrm{Nb}_{3} \mathrm{Sn}$, but at larger apertures $\left(r_{\mathrm{i}}>50 \mathrm{~mm}\right)$.

\section{IRON EFFECT}

An iron yoke has the main function of closing the magnetic circuit, thus increasing the magnetic field produced for the same current density. This means that also the magnet critical current $j_{c}$ is reduced because the load line hits the critical surface at higher field. The gradient and the peak field are assumed to be linear functions of the current $j$, i.e. we neglect saturation. At first order one can expect that the gradient and the peak field will have a similar relative increase when an iron yoke is added. In Fig. 6 we plot the difference between the relative increase in the peak field $\Delta B_{\mathrm{p}} / B_{\mathrm{p}}$ and the relative increase of the gradient in the aperture $\Delta G / G$, normalized to $\Delta G / G$, for different ratios $w / r_{i}$ and collar thickness. The increment of the gradient in the aperture $\Delta G$ has been analytically derived using the formulae of a sector coil with constant current density and iron screen, whereas the increment of the field $\Delta B_{\mathrm{p}}$ has been computed through the numerical code ANSYS. It can be stated that

- The 1st order approximation $\Delta B_{\mathrm{p}} / B_{\mathrm{p}}=\Delta G / G$ is correct within $10 \%$ in a large part of the domain of interest $0.5<$ 


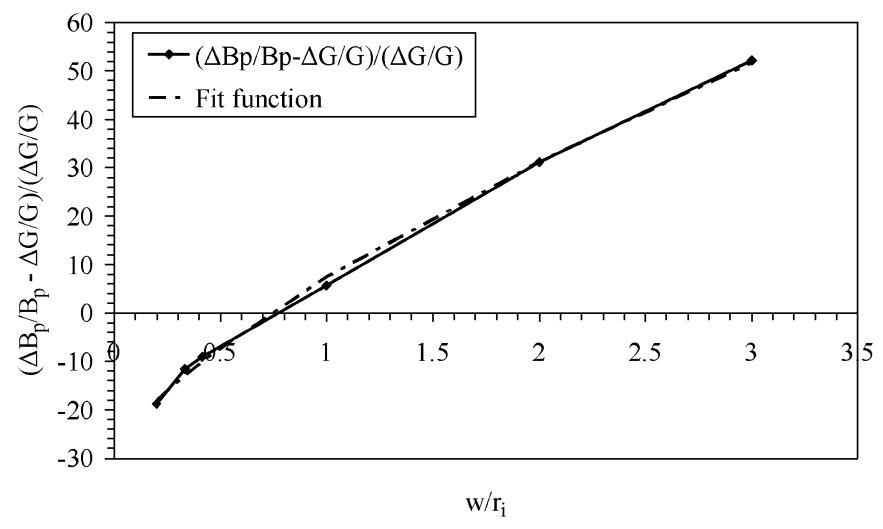

Fig. 6. Relative difference (in \%) between the relative increment of $B_{p}$ and $G$ versus $w / r_{i}$.

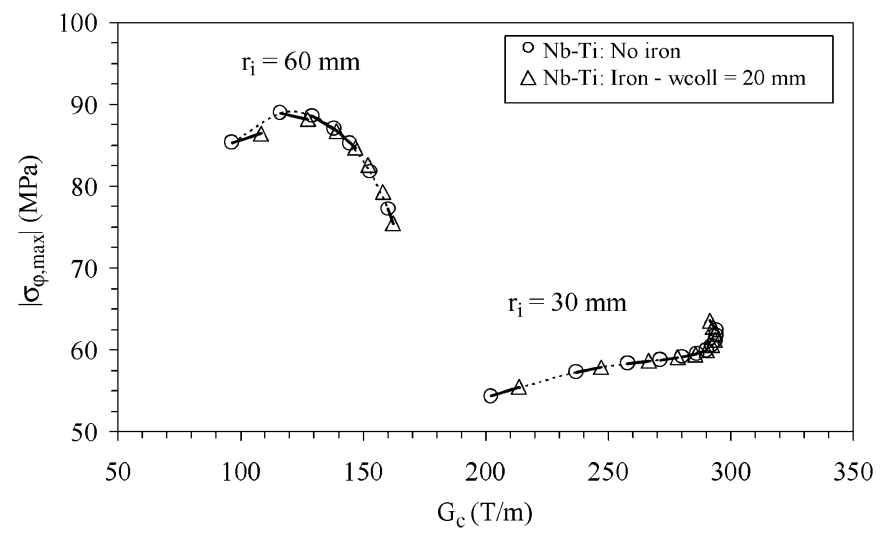

Fig. 7. Evolution of $\sigma_{\varphi, \max }$ for a collar width $w_{\text {coll }}=20 \mathrm{~mm}$, Nb-Ti case. Solid lines refer to the same coil width, varying only the iron radius.

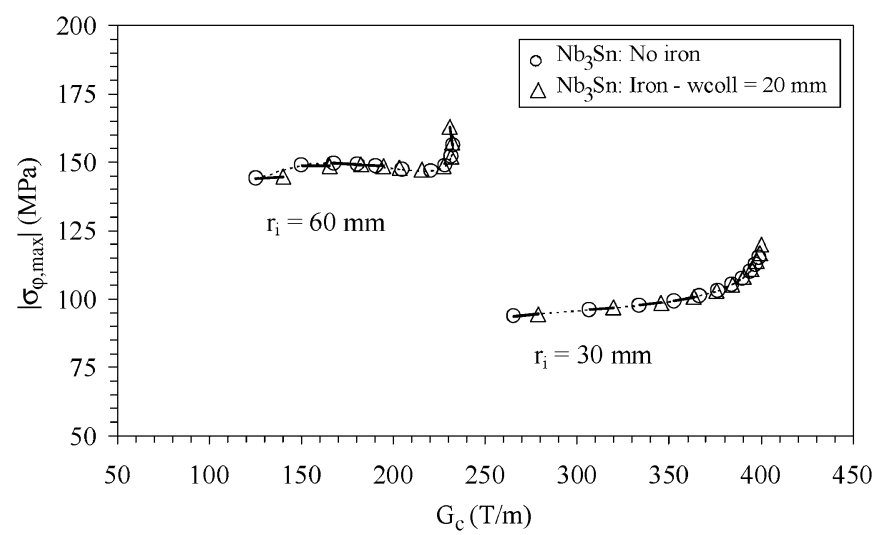

Fig. 8. Evolution of $\sigma_{\varphi, \max }$ for a collar width $w_{\text {coll }}=20 \mathrm{~mm}, \mathrm{Nb}_{3} \mathrm{Sn}$ case. Solid lines refer to the same coil width, varying only the iron radius.

$w / r_{i}<1.5$. Since this difference has to be applied on an increment, representing therefore a small error.

- The relative difference mainly depends on the ratio $w / r_{i}$, and is practically independent on the distance between the coil and the iron (i.e. the collar width).
The curve in Fig. 6 has been empirically fitted with

$$
\frac{\Delta B_{p}}{B_{p}}-\frac{\Delta G}{G}=\frac{\Delta G}{G}\left[p_{0}+p_{1}\left(\frac{w}{r}\right)^{-q}\right]
$$

with $\mathrm{p}_{0}=30.0, \mathrm{p}_{1}=37.4$, and $\mathrm{q}=0.71$. Having an analytical estimate of $\Delta G$ (given by the analytical model), and an empirical fit giving $\Delta B_{\mathrm{p}}$, we can estimate through (8) and (10) the new critical current density with the iron $j_{c, I r o n}$. This new current density can then be used together with the formulae of the forces to evaluate the maximum stress on the mid-plane. Results are shown in Figs. 7 and 8, for two apertures. The increment in gradient is followed by a variation of the peak stress that brings the new structure to the same maximum stress condition as a coil generating the same gradient in air.

\section{CONCLUSIONS}

We analysed a simplified model of superconducting quadrupole, namely a $30^{\circ}$ sector coil with uniform current density. We outlined a semi-analytical approach that allow to predict the stress distribution along the mid-plane with good precision, and that holds also for more realistic non-isotropic materials. We used $j_{\mathrm{c}}$ deduced in [2] in the previous derived equations, to show that the stress increases for larger quadrupole apertures and that the dependence on the coil thickness is more involved. For the Nb-Ti the stress is below $100 \mathrm{MPa}$ for apertures radii smaller than $60 \mathrm{~mm}$; for the $\mathrm{Nb}_{3} \mathrm{Sn}$ the stress in aperture radii smaller than $60 \mathrm{~mm}$ is below $150 \mathrm{MPa}$, which is taken as guideline for $\sigma_{\varphi, \max }$ before degradation of the superconducting properties of the material.

Introducing an iron screen, both the critical gradient and the peak stress increases to reach the same level as it would have in an ironless larger coil creating the same gradient. So further studies can be accomplished on coil in air (easier to treat analytically) since the peak stress depends only on $G_{\mathrm{C}}$.

\section{REFERENCES}

[1] E. Barzi et al., "Sensitivity of Nb3Sn Rutherford-type cables to transverse pressure," in ASC'04, IEEE Trans. Appl. Supercond., June 2005, vol. 15 , no. 2, p. 1541.

[2] E. Todesco and L. Rossi, "An estimate of the maximum gradient in superconducting quadrupoles," IEEE Trans. Appl. Supercond., this conference, submitted for publication.

[3] R. Meuser, Magnetic Field for a Thick $\cos n \theta$ Winding Engineering Note M5254, Lawrence Berkeley National Lab., 1978.

[4] R. Meuser, Stresses in a Thick $\cos \mathbf{n} \theta$ Winding Engineering Note M5256, Lawrence Berkeley National Lab., 1978.

[5] A. Asner, Cylindrical Aperture Multipoles With Constant Current Density Sector Windings CERN Internal Report, SI/Note MAE/69-15, (7-10-1969).

[6] S. Caspi and P. Ferracin, "Limits of Nb3Sn accelerator magnets," in Particle Accelerator Conference, 2005, pp. 107-111.

[7] S. Caspi, P. Ferracin, and S. Gourlay, "Graded high field Nb3Sn dipole magnets," in 19th Magnet Technology Conference, IEEE Trans. Appl. Supercond., 2006, pp. 354-357.

[8] L. Summers et al., "A model for the prediction of $\mathrm{Nb}_{3} \mathrm{Sn}$ critical current as a function of field, temperature, strain and radiation damage," IEEE Trans. Appl. Supercond, vol. 27, pp. 2041-2044, 1991.

[9] F. M. Asner, High Field Superconducting Magnets. : Oxford Science Publications, 1999, pp. 132-141, 155-159. 